\title{
Utilização de plasma de cavalo no teste de coagulase em Estafilococos isolados de leite cru
}

\section{Utilization of horse plasma in coagulase test for Staphylococci isolated from raw milk}

\author{
Luiz Henrique Cabral da Silva ${ }^{1}$; Alexandre Amorim Monteiro'; Vanerli Beloti2*; \\ Márcia de Aguiar Ferreira Barros ${ }^{3,4}$; Luís Augusto Nero; \\ Elsa Helena Walter de Santana ${ }^{4}$; Luciane Bilia de Moraes ${ }^{4}$
}

Resumo

Estafilococos têm grande importância na indústria de alimentos por serem associados a surtos de
intoxicação alimentar, pela ação de suas enterotoxinas. A produção dessas substâncias tem alta correlação
com a produção da enzima coagulase, que é detectada na identificação de cepas potencialmente
patogênicas. Esse teste é tradicionalmente realizado com plasma de coelho liofilizado ou pela sua separação
de sangue coletado de coelhos. O plasma liofilizado tem alto custo e a punção cardíaca, além de ser
bastante cruenta, permite a obtenção apenas de pequenos volumes de material. Este trabalho tem como
objetivo verificar a viabilidade da utilização de um material alternativo ao plasma de coelho, neste caso
o plasma de cavalo, no teste de coagulase para estafilococos isolados de leite cru. Assim, 410 colônias
típicas e atípicas de estafilococos isoladas de leite cru foram submetidas ao teste de coagulase utilizando-
se os dois plasmas. Os resultados identificaram $284(69,26 \%)$ colônias coagulase positivas e 126 (30,73\%)
coagulase negativas, com concordância de $100 \%$ entre os dois plasmas. Os resultados obtidos indicam
que a utilização do plasma de cavalo pode ser uma alternativa viável na pesquisa de estafilococos
coagulase positivos em leite cru. Palavras-chave: Leite, Estafilococos, teste de coagulase, metodologia

\begin{abstract}
Staphylococci are frequently related to food poisoning outbreaks that are caused by their enterotoxins. The enterotoxins production is highly correlated to coagulase production, which one is detected in identification of pathogenic strains. Usually the coagulase test is conducted using lyophilized rabbit plasma, or even fresh rabbit blood, separated by sedimentation. The lyophilized plasma has high costs and the fresh material is obtained in small volumes from the animals, what made it unpractical. The objective of this work was evaluating the utilization of an alternative material to rabbit plasma, in this
\end{abstract}

1 Aluno de Graduação de Medicina Veterinária, UEL, Londrina PR.

2 Docente do Departamento de Medicina Veterinária Preventiva, UEL, Londrina PR.

3 Médica Veterinária, responsável pelo Laboratório de Inspeção de Produtos de Origem Animal, DMVP, CCA, UEL, Londrina PR.

4 Programa de Pós-Graduação em Ciência Animal, DMVP, CCA, UEL, Londrina PR.

5 Programa de Pós-Graduação em Ciência de Alimentos, FCF, USP, São Paulo SP.

* Autor para correspondência: Beloti, V. Laboratório de Inspeção de Produtos de Origem Animal, Depto. de Medicina Veterinária Preventiva, Centro de Ciências Agrárias, Universidade Estadual de Londrina, Caixa Postal 6001, CEP 86051 990, Londrina PR Brasil. Telefone: 4333714708 -Fax: 433371 4714. E-mail: neli@sercomtel.com.br 
case the horse plasma, in coagulase test for staphylococci isolated from raw milk. So, 410 typical and atypical staphylococci colonies were tested for coagulase production using both plasmas. The results showed $284(69.26 \%)$ positives for coagulase production and $126(30.73 \%)$ negatives, with $100 \%$ of concordance between both plasmas. These results indicate the viability in using horse plasma in coagulase test for staphylococci isolated from raw milk, and complementary studies are necessary with strains from different types of food.

Key words: Milk, Staphylococci, coagulase test, methodology

\section{Introdução}

Estafilococos são importantes causadores de intoxicações alimentares, devido à produção de enterotoxinas e por possuírem alta prevalência (ZECCONI; HAHN, 2001; FRANCO; LANDGRAF, 1996). Segundo Mossel e Netten (1990), mais de 50\% das linhagens de Staphylococcus aureus são enterotoxigênicas.

O homem e os animais são os principais reservatórios de $S$. aureus, sendo a cavidade nasal seu principal habitat (WONG; BERGDOLL, 2002; FRANCO; LANDGRAF, 1996). Portadores nasais e manipuladores de alimentos com mãos ou braços que apresentem feridas infectadas com $S$. aureus são considerados importantes fontes de contaminação em alimentos (ASPERGER, 1995; LANCETTE; TATINI, 1992).

A alta incidência de $S$. aureus como agente de mastites em rebanho leiteiro torna essa infecção importante epidemiologicamente, estando relacionada à presença deste microrganismo no leite (CENCIGOGA et al., 2003; WONG; BERGDOLL, 2002; ZECCONI; HAHN, 2001; SILVA et al., 2000). Esse microrganismo é o principal agente responsável pelo aumento na contagem de células somáticas no leite, o que acarreta redução na produtividade e comprometimento na composição nutricional e microbiológica do leite (ASPENGER, 1995).

As intoxicações alimentares causadas por estafilococos são originadas da ingestão das enterotoxinas produzidas por estes microrganismos, que ocorre principalmente nos alimentos antes do consumo (WONG; BERGDOLL, 2002; FRANCO; LANDGRAF, 1996). Essas enterotoxinas são termoestáveis e não são, portanto, destruídas durante o processamento térmico dos alimentos, como a pasteurização do leite (WONG; BERGDOLL, 2002). Por essa razão, a pesquisa da presença desses microrganismos em alimentos crus é de grande importância. Estima-se que, quando presentes em contagens superiores a $10^{5}-10^{6} \mathrm{UFC} / \mathrm{ml}$ ou gr de alimento, os estafilococos produzem enterotoxinas em quantidade suficiente para induzir sintomas (EVENSON et al., 1988).

A sintomatologia e o período de incubação variam com a sensibilidade individual, quantidade de toxina e do alimento ingeridos (LOIR; BARON; GAUTIR, 2003; FRANCO; LANDGRAF, 1996). Os principais sintomas são náuseas, vômito, cãibras abdominais e diarréia. Quadros febris são incomuns e quando presentes normalmente estão associados à ingestão de grandes quantidades de toxina. O período de incubação varia de 30 minutos a 8 horas, com média entre 2 e 4 horas (WONG; BERGDOLL, 2002; DINGES; ORWIN; SCHLIEVERT, 2000; FRANCO; LANDGRAF, 1996).

Os alimentos mais relacionados a surtos de intoxicação por enterotoxinas estafilocócicas são aqueles que requerem muita manipulação no preparo e que permanecem em temperatura ambiente por longos períodos. De maneira geral os alimentos mais envolvidos nessas enfermidades são leite e derivados, cremes, tortas recheadas com creme, salada de batata, atum, frango, presunto, carnes e produtos a base de ovos (FRANCO; LANDGRAF, 1996; BREWER, 1991).

Dados acerca de toxinfecções alimentares no Brasil são escassos (FRANCO et al., 2003), entretanto acredita-se que as intoxicações por enterotoxinas estafilocócicas são muito comuns (PEREIRA et al., 
1994). Em alguns estados, entretanto, existe uma política de monitoramento dessas enfermidades. No Paraná, por exemplo, $53 \%$ dos surtos de doenças transmitidas por alimentos são causados por bactérias, sendo o leite e seus derivados representados por $11 \%$ desta parcela; os microrganismos mais importantes nestes surtos foram Salmonella spp. e $S$. aureus, com participação de $56,1 \%$ e $31,2 \%$, respectivamente (PARANÁ, 2005). No Estado de São Paulo entre 2001 e 2002 foram notificados 25 surtos de intoxicação por enterotoxina de $S$. aureus envolvendo 200 pessoas (SÃO PAULO, 2005).

Nos Estados Unidos, $20 \%$ a $40 \%$ dos surtos de toxinfecção alimentar são causados por intoxicação estafilocócica (ANDERSON et al., 2004). A presença de $S$. aureus em queijos varia entre 42 a $74 \%$, revelando assim um grande risco à saúde pública (ALMEIDA FILHO; NADER FILHO, 2000; MENDES; NUMERIANO; COELHO, 1999). Recentemente no Japão, 13.420 pessoas foram envolvidas em um surto de intoxicação ao consumirem leite desnatado contaminado com enterotoxina produzida por estafilococos (MILKPOINT, 2005). Na França, entre 1999 e 2000, $S$. aureus foi apontado como o segundo maior responsável por casos de intoxicação alimentar (LOIR; BARON; GAUTIR, 2003).

Já foi relatada a produção de enterotoxinas por outras espécies de estafilococos ( $S$. intermedius e S. hyicus), porém os surtos de intoxicação alimentar estão relacionados apenas a contaminação de alimentos por S. aureus enterotoxigênicos (ZOLI; NEGRETE; OLIVEIRA, 2002). A participação dessas outras espécies em surtos de intoxicações alimentares requer estudos aprimorados, bem como análises das condições necessárias para produção da toxina, associando todas estas informações a epidemiologia da intoxicação.

Várias provas para identificação de estafilococos foram desenvolvidas tendo como princípio a detecção de algumas enzimas. Shulz (1988) e Quinn (1994) apontam a coagulase como a primeira linha de defesa dessas bactérias estando associada com sua patogenicidade. A detecção da produção de coagulase pode ser considerada como a melhor prova para detecção de estafilococos enterotoxigênicos, devido a sua alta correlação com a produção de enterotoxinas, sensibilidade, confiabilidade e baixo custo (JASPER; INFANTE; DELLINGER, 1985). Desta forma, o teste de coagulase em tubo com plasma de coelho é o teste mais utilizado para diferenciar as amostras de estafilococos potencialmente patogênicas das inócuas.

Entretanto, o plasma liofilizado comercial possui um preço relativamente alto e a extração do plasma diretamente de coelhos tem apresentado dificuldades quanto a sua obtenção, tanto pela técnica (punção intracardíaca), como pelo grande número de animais necessários em biotério. A utilização de plasma de outros animais já foi relatada como alternativa a utilização de plasma de coelho. Adesyun e Shehu (1985) demonstraram que a identificação do gênero Staphylococcus de origem alimentar poderia ser realizada a partir da coagulação de plasmas de diferentes animais. O plasma de cavalo já foi utilizado na análise de estafilococos em carne (GIL; DURÃO, 2000).

Este trabalho teve como objetivo comparar o desempenho do plasma de cavalo e de coelho na prova de detecção da enzima coagulase produzida por estafilococos isolados de leite cru.

\section{Material e Métodos}

\section{Obtenção de cepas de Staphylococcus spp.}

Foram analisadas 410 cepas de Staphylococcus spp. obtidas de diferentes amostras de leite cru refrigerado, colhidas em propriedades da região de Pelotas-RS e Londrina-PR. As cepas de estafilococos foram semeadas em Ágar Baird-Parker para isolamento. Após incubação a $35^{\circ} \mathrm{C}$ por $48 \mathrm{~h}$, colônias típicas (negras com duplo halo) e atípicas (negras e de morfologia variada) foram estocadas em ágar nutriente e mantidas sob refrigeração. 


\section{Obtenção do plasma}

Os sangues de cavalo e coelho foram obtidos no Biotério e Hospital Veterinário da Universidade Estadual de Londrina. Dos cavalos, o sangue foi coletado por punção intravenosa e dos coelhos por punção intracardíaca. O processamento desse material foi o mesmo em ambas espécies, coletados em frascos contendo citrato a 2\%, na proporção 1:5. Após coleta, o material foi homogeneizado e centrifugado a $2000 \mathrm{rpm} / 15 \mathrm{~min}$ com retirada do sobrenadante (plasma integral) e congelado a $-18^{\circ} \mathrm{C}$ (KANTEK, 1980).

\section{Teste da coagulase}

As cepas de estafilococos estocadas foram semeadas em caldo BHI, com incubação a $35^{\circ} \mathrm{C} /$ 24h. De cada cultura, $0,1 \mathrm{ml}$ de BHI foi inoculado em $0,5 \mathrm{ml}$ de plasma de coelho e $0,5 \mathrm{ml}$ de plasma de cavalo, com incubação a $35^{\circ} \mathrm{C}$ e leituras a cada 2 horas (nas seis primeiras horas de incubação) e ao final de 24 horas. Foram consideradas coagulase positivas as culturas que apresentaram formação de coágulo, independente do grau. Controles positivo e negativo foram realizados em cada ocasião de semeadura.

\section{Resultados e Discussão}

Das 410 colônias testadas, 284 apresentaram resultado positivo na prova de coagulase com plasma de coelho e de cavalo.

Tabela 1. Resultados das leituras da prova de coagulase utilizando-se plasmas de cavalo e coelho (modelo probabilístico para comparação de teste diagnóstico, proposto por Gart e Buck (1996)

\begin{tabular}{lccc}
\hline & \multicolumn{3}{c}{ Plasma Coelho } \\
\cline { 2 - 4 } Plasma Cavalo & Positivo & Negativo & Total \\
\hline Positivo & 284 & 0 & 284 \\
Negativo & 0 & 126 & 126 \\
\hline Total & 284 & 126 & $\mathbf{4 1 0}$ \\
\hline
\end{tabular}

A análise estatística realizada (GART; BUCK, 1996) revelou um coeficiente de concordância de $100 \%$ o que representa uma correlação perfeita entre os resultados obtidos com os dois plasmas estudados. Esses resultados confirmam a possibilidade da utilização de plasma de cavalo, na prova de coagulase para identificação de Staphylococcus spp isolados de leite cru. Em estudo realizado por Adesyun e Shehu (1985), o plasma de cavalo foi um dos que apresentou maior correlação com o plasma de coelho para análise de coagulase.

Assim, a possibilidade de utilizar plasma de cavalo em substituição ao de coelho pode auxiliar muitos laboratórios de análises clínicas e de alimentos, além de instituições de pesquisas, significando uma redução em seus custos. Além disso, sua obtenção é muito mais fácil, prática e menos cruenta, favorecendo o bem estar animal.

\section{Conclusões}

A identificação da produção da enzima coagulase por estafilococos, através da prova de coagulase em tubos, utilizando o plasma de cavalo pode substituir, com grande confiança, o teste de coagulase tradicional feito com o plasma de coelho, representando uma importante alternativa. Estudos complementares com estafilococos isolados de outros alimentos são importantes para confirmação da viabilidade do uso do plasma de cavalo na prova de coagulase, para identificação de estafilococos de outras origens.

\section{Referências}

ADESYUN, A. A.; SHEHU, L. M. Detection of staphylocoagulase using plasmas from various animals. Veterinary Microbiology, Amsterdam, v.10, n. 4, p.387-392, 1985.

ALMEIDA FILHO, E. S.; NADER FILHO, A. Ocorrência de S. aureus em queijos tipo frescal. Revista de Saúde Pública, São Paulo, v.6, n.34, p.578-80, 2000.

ANDERSON, J. B.; SHUSTER, T. A.; HANSEN, K. E.; LEVY, A. S.; VOLK, A. A camera's view of consumer foodhandling behaviors. Journal of the American Dietetic Association, Chicago, v.104, n.2, p.186-191, 2004. 
ASPENGER, H. Staphylococcus aureus In: IDF GROUP OF EXPERTS A10/11 The significance of pathogenic microorganisms in raw milk. $2^{\text {th }}$ ed. Brussels: International Dairy Federation, 1995. p.24-43.

BREWER, M. S. Food storage, food spoilage, and foodborne illness. Urbana: University of Illinois at Urbana-Champaign, 1991. 19 p. (Circular 1313).

CENCI-GOGA, B. T.; KARAMA, M.; ROSSITTO, P. V.; MORGANETE, R. A.; CULLOR, J.S. Enterotoxin production by S. aureus isolated from mastitic cows. Journal of Food Protection, Des Moines, v.66, n.9, p.1693-96, 2003.

DINGES, M. M.; ORWIN,P.M.; SCHLIEVERT,P.M. Exotoxins of Staphylococcus aureus. Clinical Microbiology Reviews, Washington, v.13, n.1, p.16-34, 2000.

EVENSON, M. L.; HINDS, M. W.; BERNSTEIN, R. S.; BERGDOLL, M. S. Estimation of human dose of staphylococcal enterotoxin A from a large outbreak of staphylococcal food poisoning involving chocolate milk. International journal of Food Microbiology, Amsterdam, v. 7, p. 311-316, 1988.

FRANCO, B. D. G. M.; LANDGRAF, M. Microbiologia dos Alimentos. São Paulo: Atheneu, 1996.

FRANCO, B. D. G. M.; LANDGRAF, M.; DESTRO, M. T.; GELLI, D. Foodborne diseases in Southern South América. In: MILIOTIS, M.; BIER, J. (Ed.). International Handbook of Foodborne Pathogens. New York: Marcel Dekker, 2003. p. 733-743.

GART, J. J.; BUCK, A. A. Comparison of a screening test and a reference test in epidemiologic studies. American Journal of Epidemiology, Baltimore, v.83, n.1, p.593-602, 1996.

GIL, J. I.; DURÃO, J. C. Manual de inspeção sanitária de carnes. Lisboa: Fundação Calouste Gulbenkian, 2000.563 p.

JASPER, D. E.; INFANTE, F.; DELLINGER, J. D. Relationships among the results as coagulase, staphylococcal toxins and thermonuclease tests on staphylococci from cow milk. Journal of Clinical Microbiology, Washington, v.21, n.4, p.582-584, 1985.

KANTEK, C. E. Elementos de patologia clínica veterinária. Curitiba: Reprocopy, 1980.

LANCETTE, G. A.; TATINI, S. R. Staphylococcus aureus In.: DOWNES, F. P.; ITO, K. (Ed.). Comnpendium of methods for the microbiological examination of foods. $3^{\text {th }}$ ed. Washington: American Public Health Association, 1992. p. 533-593.

LOIR, Y.; BARON, F.; GAUTIR, M. Staphylococcus aureus and food poisoning. Genetic Molecular Research, Rennes, v.2, n.1, p.63-76, 2003.
MENDES, E. S.; NUMERIANO, A M.; COELHO, M. I. S. S. Aureus, Salmonella sp e coliformes em queijos de coalho comercializado em Recife. Revista Higiene Alimentar, São Paulo, v.13, n.66, p.122-126, 1999.

MILKPOINT. Leite com alta CCS pode significar risco à saúde humana. Disponível em http:// www.milkpoint.com.br. Acesso em: jun 2005.

MOSSEL, D. A. A.; NETTEN, VAN P. Staphylococcus aureus and related staphylococci in food: ecology, proliferation, toxigenesis, control and monitoring. Journal of Applied Microbiology, Oxford, v.69, p. 235-1459, 1990.

PARANÁ. Secretaria da Saúde do Estado do Paraná. Saúde Paraná. Disponível em http://www.saude.pr.gov.br. Acesso em: 15 mar. 2005.

PEREIRA, M. L.; CARMO, L. S.; SANTOS, E. J.; BERGDOLL, M. S. Staphylococcal food poisoning from cream-filled cake in a metropolitan area. Revista de Saúde Pública, São Paulo, v.28, n.6, p.406-409, 1994.

QUINN, N. E. Clinical veterinary microbiology. London: Wolfe, 1994.

SÃO PAULO. Secretaria da Saúde do Estado de São Paulo. Staphylococcus Aureus e intoxicação alimentar. Disponível em http://www.saude.sp.gov.br. Acesso em: 15 mar. 2005.

SHULZ, W. Infecções por estafilococos. In: BEER, J. Doenças infecciosas dos animais domésticos. São Paulo: Roca, 1988. p.1-9.

SILVA, W.P.; DESTRO, M. T.; LANDGRAF, M.; FRANCO, B. D. G. M. Biochemical characteristics of typical and atypical Staphylococcus aureus in mastitic milk and environmental samples of brazilian dairy farms. Brazilian Journal of Microbiology, São Paulo, v.31, n.2, p.103-106, 2000.

WONG, A. C. L.; BERGDOLL, M. S. Staphylococcal Food Poisoning. In: CLIVER, D.; RIEMANN, H. Foodborne diseases. $2^{\text {th }}$ ed. Amsterdam: Academic Press, 2002. p. 231-248.

ZECCONI, A.; HAHN, G. Staphylococcus aureus in raw milk and human health risk. Bulletin of IDF, Brussels, v.345, p.15-18, 2001.

ZOLI, J. A.; NEGRETE, I. R. A.; OLIVEIRA, T. C. R. M. Avaliação da contaminação por $S$. aureus e Salmonella spp., de maionese de batata comercializada em Londrina, PR. Higiene Alimentar, São Paulo, v.16, n.95, p.62-70, 2002. 